
ISSN: 2576-0971

journalbusinessentrepreneurial@gmail.com

Colloquium editorial

Ecuador

\title{
Planeacion estrategica para el desarrollo sustentable en el sector turistico
}

Rivera Guerrero, Álvaro

Planeacion estrategica para el desarrollo sustentable en el sector turistico

Journal of business and entrepreneurial studies, vol. 3, núm. 2, 2019

Colloquium editorial, Ecuador

Disponible en: http://www.redalyc.org/articulo.oa?id=573660685003

DOI: https://doi.org/10.31876/jbes.v3i2.22

Esta obra está bajo una Licencia Creative Commons Atribución-NoComercial-SinDerivar 4.0 Internacional. 


\title{
Planeacion estrategica para el desarrollo sustentable en el sector turistico
}

Strategic planning for sustainable development in the tourism

sector

\author{
Álvaro Rivera Guerrero alvaro.rivera@gmail.com \\ Universidad Cooperativa de Colombia, Universidad Libre - Campus Cali, \\ Colombia
}

http://orcid.org/0000-0001-8786-9451

Journal of business and entrepreneurial studies, vol. 3, núm. 2, 2019

Colloquium editorial, Ecuador

Recepción: 03 Diciembre 2018 Aprobación: 20 Enero 2019

DOI: https://doi.org/10.31876/ jbes.v3i2.22

Redalyc: http://www.redalyc.org/ articulo.oa?id= 573660685003

\section{BY-NC-ND}

Resumen: La historia de la humanidad esta llenas de ejemplos de civilizaciones que en la cúspide de su desarrollo abruptamente caen, y pronto desaparecen. Aunque al respecto se ha dicho mucho y las razones de su extinción se atribuyen a muchas causas, en general se puede afirmar que no obstante las organizaciones sociales de producción y trabajo (civilizaciones) logran con éxito el desarrollo sostenido (aquel que por sus propias fuerzas y lógica se reproduce asimismo), no logran el desarrollo sustentable (aquel que prevé las necesidades del crecimiento futuro). Por lo anterior, dada la experiencia histórica, para las civilizaciones actuales de occidente y de oriente, la cuestión de interés es saber cómo hacer que lo sostenible sea sustentable, siendo que la lógica de acumulación capitalista implica para ello considerar aspectos que son sustento de su existencia y reproducción. El turismo en Ecuador debe convertirse en un sector altamente competitivo, por medio de un proceso articulado de inversión y promoción, con productos y servicios, que en cantidad y calidad suficientes se traduzcan en crecimiento y desarrollo social para hacer posible esto se requiere de la conjunción de esfuerzos de los tres niveles: local, nacional e internacional.

Palabras clave: Turismo, Inversión, Sostenible, Competitivo, Desarrollo.

Abstract: The history of humanity is full of examples of civilizations that at the peak of their development abruptly fall, and soon disappear. Although about much has been said and the reasons for their extinction is attributed to many causes, in general we can say that despite the social organizations of production and work (civilizations) achieve successful sustainable development (who by their own forces and logic is also playing) do not achieve sustainable development (provided that the needs of future growth). Therefore, given the historical experience for current civilizations of the West and the East, the question of interest is how to make it sustainable is sustainable, being that the logic of capitalist accumulation means for it to consider aspects that are supporting his existence and reproduction. Tourism in Ecuador should become a highly competitive sector, through an articulated investment process and promotion, products and services, which in sufficient quantity and quality will translate into growth and development social to make this possible requires the combined efforts of the three levels: local, national and international.

Keywords: Tourism, Investment, Sustainable, Competitive Development.

\section{Introducción}

La Organización Mundial de Turismo, (2012) manifiesta que: la contribución del turismo a laactividad económica mundial se estima en cerca del $5 \%$ de las exportaciones globales debienes y servicios ubicándose en cuarto lugar en relación con la exportación de servicios. Elturismo representa el $30 \%$ del total de ingresos, cuya contribución al empleo 
tiende a serligeramente superior en términos relativos y se estima entre el $6 \%$ y el $7 \%$ del número total deempleos en todo el mundo (directo e indirecto).

En el Ecuador el turismo es el cuarto sector productivo que contribuye al PIB del país luego de la construcción, la agricultura-pesca, el turismo y la industria manufacturera. El turismo, por su parte, es una actividad bastante dinámica. Según cifras del Ministerio deTurismo, (2012) la actividad ha crecido a un $12 \%$, superando de largo a la tendencia generalen el mundo que lo ha hecho en aproximadamente $4 \%$. Incluso, según la misma entidad, esteaño se registraron picos importantes de crecimiento, llegando incluso al $20 \%$ a inicios de año.A pesar de mantener un crecimiento en número de visitantes y aportes al PIB, el turismo en elEcuador todavía no alcanza una participación en la economía paralelo al contexto mundial

Ante esta realidad, en el PLANDETUR 2020 se asevera que: el Ecuador necesita undesarrollo turístico sostenible que cuide nuestra riqueza histórico-cultural, el medio ambiente,las comunidades y reactive la economía.

La presente investigación formula el Plan estratégico de desarrollo turístico sostenible del cantón Paltas, provincia de Loja; que se inició con el diagnóstico situacional del mismo, el cual permitió obtener una clara perspectiva de la realidad actual que atraviesa el Cantón.

A continuación se realizó el análisis del potencial turístico del cantón Paltas, por medio de la visita In-Situ (Agosto 2012), en la que se hiso el análisis de la oferta (Caracterización de los productos turísticos, uso de TIC's) y demanda (perfil del visitante e identificación de la problemática turística) por medio de entrevistas, encuestas y el análisis FODA de todos los componentes relacionados a la actividad turística, a partir de este estudio se planteó la formulación estratégica (objetivos del plan estratégico, proceso metodológico y elaboración de estrategias) priorizando las estrategias más relevantes, con las mismas se plantearon cuatro programas y quince proyectos, cuya duración horizonte es de 5 años, finalmente se realizó la formulación filosófica (visión, misión, valores), para posicionar a Paltas como destino turístico, mejorar la calidad de vida de sus habitantes y contribuir al desarrollo económico del mismo.

Es por ello que, en estos últimos años Ecuador ha incluido al turismo sostenible dentro de lapolítica de Estado, buscando diversificar la economía, asegurar igualdad de oportunidades degénero, conservar el ambiente, mejorar la calidad de vida, empoderar y conservar ladiversidad de culturas del país

El Ecuador es un país que posee un gran potencial turístico ya que es uno de los 17 países donde se halla concentrada la mayor biodiversidad del planeta, en su interior se encuentran habitando 13 nacionalidades indígenas, existe una gran diversidad en producción artesanal en cuero, joyería, textiles, cerámica, fibras vegetales; que se ve complementada con la existencia de ciudades impresionantes que constituyen patrimonio de 
la Humanidad y con la belleza paisajística en cada una de las regiones naturales.

La actividad turística en nuestro país en el transcurso de los años se ha consolidado hasta constituirse en el tercer producto de exportación, el mismo que genera divisas para varios sectores de la economía local, constituyéndose en una excelente alternativa para la generación de ingresos de los diversos asentamientos humanos a lo largo del territorio de nuestra patria, convirtiéndose conjuntamente en una herramienta para la reducción de la pobreza y la generación de empleo.

El Cantón Paltas es uno de los cantones mayormente reconocidos a lo largo de la provincia de Loja, encontrándose en su interior gran biodiversidad y una infinidad de atractivos turísticos que van complementados con una amplia infraestructura y planta turística, la misma que ha sido difundida a nivel nacional e internacional buscando brindar las condiciones más adecuadas para el mayor disfrute del entorno.

Los Planes Estratégico de Desarrollo Turístico Sostenible para el Cantón Paltas Provincia de Loja, pretende que mediante la formulación de programas y proyectos articulados con los lineamientos del Plan Estratégico de Desarrollo Turístico del cantón, logre consolidar al cantón Paltas como un destino turístico nacional e internacional, fomentandocon ello el aprovechamiento sostenible de los recursos naturales, culturales y generando nuevas fuentes para la obtención de ingresos económicos.

La elaboración de un plan para el desarrollo del turismo sostenible implica un proceso participativo con los sectores público, privado y comunitario, organizaciones no gubernamentales y entre otros actores. $\mathrm{El}$ proceso requiere el desarrollo de varias etapas, la primera etapa consiste en el diagnóstico del cantón en cual se analizarán las condiciones físicas, espaciales, ambientales, económicas, productivas, sociales y culturales del territorio permitiendo de esta manera tener una visión completa del cantón; la segunda etapa requiere el desarrollo de un estudio de mercado para tener un análisis de la oferta territorial, demanda y competencia turística; la tercera etapa plantea la visión filosófica del plan y el análisis de la problemática que incide en el desarrollo turístico en los diferentes niveles que conforman el sistema para finalmente formular el plan estratégico de desarrollo turístico sostenible.

\section{Materiales y métodos}

El turismo es uno de los sectores fundamentales en el crecimiento económico de la mayor parte de territorios que abarcan la República del Ecuador, su incremento o disminución se halla ligado a diversos ámbitos tales como: sociales, psicológicos, económicos, entre otros; los mismos que inciden directa o indirectamente, razón por lo cual es de vital importancia tomar las medidas necesarias que permitan llevar un control y monitoreo de la actividad turística que mediante la formulación de acciones permita contrarrestar las falencias existentes y a su vez ir 
mejorando las condiciones territoriales y de servicios para procurar el desarrollo de un turismo sostenible y sustentable.

El Gobierno Autónomo Descentralizado Municipal Paltas ha considerado como una de sus actividades productivas el turismo y con el propósito de facilitar el desarrollo de esta actividad, consolidar al territorio como un destino turístico competitivo, aprovechar los recursos naturales y culturales de forma sostenible ha visto conveniente la formulación del Plan Estratégico de Desarrollo Turístico Sostenible, el mismo que constituirá una herramienta eficaz para orientar la inversión pública y mejorar la calidad de vida de sus habitantes.

El Plan Estratégico de Desarrollo Turístico Sostenible realizará de forma participativa con la finalidad de lograr una profunda reflexión en relación a la identificación de factores que inciden negativamente en el desarrollo del turismo y posteriormente plantear las mejores soluciones a corto, mediano y largo plazo que permitan innovar la oferta turística actual como una estrategia sostenible de contribución al bienestar colectivo.

Como objetivo de la investigación y promover al turismo es de "Diseñar un Plan Estratégico de Desarrollo Turístico Sostenible del Cantón Paltas Provincia de Loja. La hipótesis que se plantea es como el Plan Estratégico de Desarrollo Turístico Sostenible consolidará al cantón Paltas como un destino turístico competitivo, en el cual se aprovechan sosteniblemente los patrimonios culturales y naturales para la generación de recursos económicos y el mejoramiento de la calidad de vida de sus habitantes.

El sistema turístico por su parte está constituido por un conjunto de elementos interdependientes que permiten su funcionamiento y que se encuentra en estrecha relación con la vida de todos los pueblos, ya sea que su involucramiento sea como turistas o como anfitriones. El turismo se estructura en cuatro elementos sustanciales a saber: los atractivos y las consiguientes actividades que éstos posibilitan, la infraestructura social, la planta turística y la superestructura.

El desarrollo sostenible puede ser definido como "un desarrollo que satisfaga las necesidades del presente sin poner en peligro la capacidad de las generaciones futuras para atender sus propias necesidades". (ONU, 2000). De acuerdo a las tres dimensiones básicas del desarrollo sostenible: bienestar económico, seguridad social y estabilidad ecológica una expresión adecuada y necesaria del desarrollo.

Utiliza al mercado como medio eficaz para la creación de bienestar, se compromete con el objetivo de la justicia social mediante normas de compensación y diseña todos los procesos económicos desde un principio, de manera que las bases de la existencia puedan ser aseguradas.

Se trata no solo de proteger el medio ambiente y naturaleza por medio de medidas defensivas que curen los síntomas, sino de convertir esta defensa en un factor al que se le apunte en el diseño del futuro, que reconozca y proteja la naturaleza en su valor propio y en su dimensión diversa.(LAPSO, DAAD, 2003).

Paltas es uno de los cantones más antiguos del País. Se erigió cantón con el nombre de Catacocha, por la Ley de división territorial en tiempos 
de la Gran Colombia el 25 de Junio de 1824, seis años antes que la República del Ecuador. A pesar de que el concejo Municipal en el año 1954, presidido por el Sr. Ezequiel Guamán, declara fecha cívica de Cantonización de Paltas el 25 de Junio, no es, hasta el año 1965, cuando se celebra por primera vez la fiesta de aniversario cantonal. En el año 1861, mediante una nueva ley de división territorial aparece con el nombre de cantón Paltas. (GAD del cantón Paltas, 2011)

\section{Resultados}

Según la Ley de Turismo en el Capítulo II de las Disposiciones Transitorias clasifica a la planta turística de la siguiente manera:

El potencial turístico, se denomina a las zonas, territorios, lugares, etc., en donde es más

Según la Ley de Turismo en el Capítulo II de las Disposiciones Transitorias clasifica a la planta turística de la siguiente manera:

El potencial turístico, se denomina a las zonas, territorios, lugares, etc., en donde es más factible el desarrollo de la actividad turística por diversos factores que facilitan y coadyuvan a que esta actividad se desenvuelva con los resultados positivos esperados.

El presente proyecto tiene como finalidad determinar los atractivos turísticos del cantón Paltas y evaluar los atractivos turísticos potenciales del mismo; dando a conocer al GAD del cantón Paltas, a los GAD Parroquiales y a la colectividad en general, la riqueza cultural y turística que posee; para que sean ellos los que promuevan y aporten al desarrollo turístico del Cantón, mediante una cultura turística apropiada que lleve a mejorar la calidad de vida de los Paltenses.

Para alcanzar esta meta se tiene que identificar y valorar los componentes del sistema turístico del cantón Paltas de acuerdo a la metodología para inventario de atractivos turísticos del MINTUR (MINTUR, 2004).

Para establecer el potencial turístico que conserva el cantón Paltas es preciso analizar los atractivos turísticos que se encuentran inventariados en el Cantón, como son: Shiriculapo, Museo de los Hermanos Maristas, Piedra del Sol, sin embargo en la visita In-Situ (Agosto 2012) al cantón Paltas se pudo realizar el levantamiento de información de algunos atractivos que por las condiciones que presentan poseen potencial turístico.

La caracterización de los Productos Turísticos del cantón Paltas se realizó una fase de diagnóstico para conocer la potencialidad turística del cantón Paltas y se analizó las siguientes fuentes de consulta:

Fuentes de información secundaria: Plan estratégico de turismo sostenible del Ecuador (PLANDETUR 2020), plan de desarrollo y ordenamiento territorial cantonal del cantón Paltas, 2011 y 2012; mapas, información sobre estadísticas y censos (INEC, 2010), Inventario de Patrimonio Cultural del cantón Paltas (INPC, 1993) catastros del MINTUR, Inventario de Atractivos Turísticos del MINTUR, 
fotografías, directorios locales y regionales; y oficinas de turismo gubernamentales tanto locales y regionales.

Fuentes de información primaria: Consultas al personal del GAD del cantón Paltas, de la oficina de Promoción de Turismo y Cultura; departamento de Avalúos y Catastros y departamento de planificación del Gobierno Autónomo Descentralizado del cantón Paltas; visita InSitu (Agosto 2012) al cantón Paltas y por ende a sus atractivos turísticos con el fin de determinar y levantar el Inventario Turístico de atractivos existentes en el cantón, que no estan inventariados puesto que la Subsecretaria Frontera Sur del Ministerio de Turismo posee información escasa de los mismos.

Fase de Formulación

Para desarrollar esta fase se trabajó con información proporcionada por la oficina de Promoción Turismo y Cultura del GAD Paltas y en la visita In- Situ (Agosto, 2012) a los sectores involucrados con el turismo del cantón Paltas.

Los productos turísticos

(Acerenza, M. Á., 2004), manifiesta que el producto turístico no es más que un conjunto de prestaciones, materiales e inmateriales, que se ofrecen con el propósito de satisfacer los deseos o las expectativas del turista.

El producto turístico puede ser analizado en función de los componentes básicos que lo integran: Atractivos, Facilidades y Accesos.

A continuación se detallan todos los componentes básicos que lo integran, los mismos que combinados entre si originan el producto final que se ofrece en el mercado al turista.

Producto desarrollado en el cantón Paltas

Analizando la línea de productos, se puede determinar que el cantón Paltas tiene desarrollado dentro del turismo cultural: El turismo religioso que se da en Catacocha todos los años entre el segundo y tercer sábado de Enero, y el 3 de mayo la romería de la Cruz del Agua Fría en donde la afluencia promedio de visitantes es de 2000 personas.

Productos que pueden desarrollarse a través de la línea de productos contemplados en el PLANDETUR 2020

Siguiendo la línea de productos del PLANDETUR 2020, se observa que otros productos se pueden desarrollar en el cantón Paltas los mismos que se describen a continuación:

Turismo comunitario, se puede potencializar en las parroquias rurales Guachanamá, San Antonio y Yamana gracias a las riquezas naturales y culturales que poseen y a los habitantes que se encuentran capacitados en actividades turísticas, para ofrecer servicios de calidad; también se puede desarrollar esta actividad en el barrio Tacoranga en donde los turistas podrán ser partícipes de la elaboración de cerámica de forma artesanal.

v Dentro del Turismo cultural, el Patrimonio natural y cultural existente que posee un gran potencial de arquitectura colonial razón por la cual la cabecera cantonal Catacocha fue declarada Patrimonio Cultural del Ecuador, el Turismo de mercado y artesanías en el barrio Tacoranga donde realizan todo tipo de artesanías en barro y algunos tejidos y el 
Turismo arqueológico por la existencia de petroglifos y petrograbados en las parroquias de Yamana, San Antonio, Lourdes.

v Dentro del Turismo de deportes y aventura, el Deporte terrestre se puede implementar Canopy desde la ciudad de Catacocha al cerro de Huanchuro, descenso y ascenso en la Peña del Shiriculapo, en las chorreras de Soracola, Consacola y Landara; que con la implementación de una excelente infraestructura pueden generar posibilidades llamativas para el turista.

v El Agroturismo, es otra de las líneas de productos que se puede desarrollar en la zona, gracias a la existencia del Centro Binacional Zapotepamba donde se puede conocer las diferentes actividades agrícolas y ganaderas desde su fase inicial hasta la elaboración de productos; tal como es el caso de la Asociación de producción de plantas medicinales La Laureñita de la parroquia Lauro Guerrero que actualmente se encuentra muy bien organizada con su producto estrella la Horchata.

Proyectos del PLANDETUR 2020 que deben ser implementados en el cantón Paltas

Una vez revisadas todas las bases estratégicas del turismo en el Ecuador, compuestas por los programas, proyectos y actividades del PLANDETUR 2020 y teniendo en cuenta que las mismas surgieron de la problemática existente en cada región del País intervenida, se determina que de los mismos para el Cantón Paltas, se debe priorizar los siguientes programas:

- Consolidación de la seguridad íntegra del turismo a través del desarrollo de proyectos

- Ordenamiento para el turismo sostenible

- Facilitación turística

- Desarrollo de destinos turísticos

Fácilmente el turismo en este cantón puede desarrollarse a plenitud y constituirse en un valioso aporte para mejorar la calidad de vida de sus habitantes, desafortunadamente sus recursos no han sido adecuadamente reconocidos, definidos y más que todo valorados por el GAD Cantonal y los GAD Parroquiales, ni por la comunidad residente en el Cantón.

El cantón Paltas ofrece un sin número de actividades como: turismo comunitario, turismo cultural y religioso, turismo arqueológico, turismo deportivo y de aventura y agroturismo.

\section{Discusión}

Es necesario convocar a distintos sectores sociales a participar de forma activa y eficiente, por medio de propuestas que permitan desarrollar programas adecuados. El desarrollo en Ecuador y particularmente en el Cantón Paltas Provincia de Loja, se verán beneficiados con tales programas. Evidentemente se requiere urgentemente de: generación de empleos, ingresos y divisas, inversión, etc. Todo ello, con respeto a nuestros valores e identidad cultural, y sobre todo y ante todo con franco y claro respeto al entorno ambiental y ecológico 
"El Turismo Sostenible atiende a las necesidades de los turistas actuales $\mathrm{y}$ de las regiones receptoras y al mismo tiempo protege y fomenta las oportunidades para el futuro. Se concibe como una vía hacia la gestión de todos los recursos de forma que puedan satisfacerse las necesidades económicas, sociales y estéticas, respetando al mismo tiempo la integridad cultural, los procesos ecológicos esenciales, la diversidad biológica y los sistemas que sostienen la vida".

El territorio que forma parte de la jurisdicción del cantón Tena cuenta con recursos naturales y culturales que pueden ser aprovechados sosteniblemente, mediante el desarrollo de la actividad turística, sin embargo, su continua degeneración es alarmante, lo cual conjugado con la deficiente dotación de servicios básicos reduce la calidad de la oferta turística y produce una disminución paulatina del nivel de satisfacción de la visita.

El cantón Paltas, cuenta con recursos naturales y culturales que pueden ser aprovechados sosteniblemente, mediante el desarrollo de la actividad turística, sin embargo, la falta de conocimiento de los atractivos con potencial turístico, provoca que se deterioren por varios factores, entre los cuales están las condiciones climáticas, el perjuicio por las personas que circulan por los lugares donde están ubicados y la falta de mantenimiento de los atractivos por las autoridades competentes.

La desvalorización de los sitios patrimoniales, y la falta de información sobre estos fomentan la pérdida de identidad de la comunidad Paltense.

La falta de diversificación de la oferta actual que ofrece el cantón Paltas, no es la adecuada para captar nuevos segmentos de mercado turístico.

\section{Conclusión}

La demanda turística en el cantón Paltas está dada por la cantidad de visitantes en feriados, fiestas de cantonización, populares y religiosas, gracias a la investigación se concluyó que debido a la falta de promoción de los atractivos naturales y culturales que posee el Cantón los turistas nacionales que visitan Paltas tienen desconocimiento de la existencia de los mismos.

En base a las encuestas realizadas se concluye que la demanda califica como buena la amabilidad y calidez de los Paltenses, pero considera que se debe mejorar la calidad en los servicios hoteleros prestados.

El Plan estratégico de desarrollo turístico sostenible para el cantón Paltas se constituye en la herramienta clave para el desarrollo turístico del cantón, la cual se sustenta por su visión y misión que se cumplirá gracias a la vinculación del sector público, sector privado y la comunidad.

El desarrollo de un proceso participativo para la construcción del plan se constituyó en el componente clave para aglutinar a los sectores privado, público y comunitario del cantón Paltas, permitiendo definir una visión conjunta y clara para el desarrollo sostenible del turismo y los objetivos estratégicos que orienten la ejecución de propuestas y actividades en los próximos cinco años. 
El Plan estratégico de desarrollo turístico sostenible del cantón Tena se constituye en la herramienta estratégica de articulación de la política turística local con la política nacional establecida en el Plan Estratégico de Desarrollo Turístico del Ecuador 2020 (PLANDETUR2020), lo cual permite que los proyectos formulados puedan contribuir a la consecución del Sumak Kawsay al ser financiados por instituciones públicas así como también por organismos de cooperación.

\section{Referencias}

Acerenza, M. Á. (2004). Fundamentos de marketing turistico (Primera ed.). . México: Trillas. Cedisa libros Primera Edición

Ayala, L. (2011). Planeación y Administración Estratégicas una herramientapara el éxito. Madrid. España Mc. Graw Hill

Caimi, M. (2003). Discurso del método. Buenos Aires, Argentina. Ediciones Colihue SRL.

Kotler, P. (2011). Marketing turístico . Madrid: Pearson

Lapso, D. (2003). Pedagogía social en Latinoamérica. Estrategias en educación popular, desarrollo e interculturalidad. Berlin, Alemania, Editorial DAAPS

Ministerio de Turismo. (2004). Guia para inventarios de atractivos turísticos .

Organización de las Naciones Unidas. (2000). Organización de las Naciones Unidas . Obtenido de "Medio ambiente y desarrollo sostenible"

Rey, M. (2004). Fundamentos de marketing turístico . Madrid. España: Síntesis.

Tobón, S. (2007). El enfoque complejo de las competencias y el diseño curricular. Acción pedagógica, (16) 14-28

Vargas, F; Casanova, F; y Montanaro, L. . (2003). El Enfoque de Competencia Laboral: Manual de Formación. J. León. (Presidencia), CINTEFOR/ OIT/2001. Citados por Miguel A. Sahagún, Montevideo. Uruguay

\section{Notas de autor}

alvaro.rivera@gmail.com 